\title{
Neonatal hydrocephalus leads to white matter neuroinflammation and injury in the corpus callosum of Ccdc39 hydrocephalic mice
}

\author{
Danielle S. Goulding, BS, ${ }^{1,2}$ R. Caleb Vogel, BS, ${ }^{1,2}$ Chirayu D. Pandya, PhD, ${ }^{1,2}$ Crystal Shula, BS, ${ }^{3}$ \\ John C. Gensel, PhD,,,4 Francesco T. Mangano, DO, ${ }^{3}$ June Goto, PhD, ${ }^{3}$ and \\ Brandon A. Miller, MD, PhD ${ }^{1,2}$ \\ ${ }^{1}$ Department of Neurosurgery, ${ }^{2}$ Spinal Cord and Brain Injury Research Center, and ${ }^{4}$ Department of Physiology, University \\ of Kentucky, Lexington, Kentucky; and 'Division of Pediatric Neurosurgery, Cincinnati Children's Hospital Medical Center, \\ Cincinnati, Ohio
}

\begin{abstract}
OBJECTIVE The authors sought to determine if hydrocephalus caused a proinflammatory state within white matter as is seen in many other forms of neonatal brain injury. Common causes of hydrocephalus (such as trauma, infection, and hemorrhage) are inflammatory insults themselves and therefore confound understanding of how hydrocephalus itself affects neuroinflammation. Recently, a novel animal model of hydrocephalus due to a genetic mutation in the Ccdc39 gene has been developed in mice. In this model, ciliary dysfunction leads to early-onset ventriculomegaly, astrogliosis, and reduced myelination. Because this model of hydrocephalus is not caused by an antecedent proinflammatory insult, it was utilized to study the effect of hydrocephalus on inflammation within the white matter of the corpus callosum.
\end{abstract}

METHODS A Meso Scale Discovery assay was used to measure levels of proinflammatory cytokines in whole brain from animals with and without hydrocephalus. Immunohistochemistry was used to measure macrophage activation and NG2 expression within the white matter of the corpus callosum in animals with and without hydrocephalus.

RESULTS In this model of hydrocephalus, levels of cytokines throughout the brain revealed a more robust increase in classic proinflammatory cytokines (interleukin [IL]-1 $\beta$, CXCL1) than in immunomodulatory cytokines (IL-10). Increased numbers of macrophages were found within the corpus callosum. These macrophages were polarized toward a proinflammatory phenotype as assessed by higher levels of CD86, a marker of proinflammatory macrophages, compared to CD206, a marker for antiinflammatory macrophages. There was extensive structural damage to the corpus callosum of animals with hydrocephalus, and an increase in NG2-positive cells.

CONCLUSIONS Hydrocephalus without an antecedent proinflammatory insult induces inflammation and tissue injury in white matter. Future studies with this model will be useful to better understand the effects of hydrocephalus on neuroinflammation and progenitor cell development. Antiinflammatory therapy for diseases that cause hydrocephalus may be a powerful strategy to reduce tissue damage.

https://thejns.org/doi/abs/10.3171/2019.12.PEDS19625

KEYWORDS myelin; corpus callosum; microglia; neuroinflammation; hydrocephalus

$\mathrm{H}$ YDROCEPHALUS can be caused by many pathological events, including intracerebral hemorrhage, trauma, and infection. Untreated hydrocephalus is often associated with progressive neurological injury, but good outcomes can be achieved with early diagnosis and CSF diversion surgery. Unfortunately, early prognostic biomarkers and efficacious medical therapies to treat hydrocephalus are lacking. There are also no guidelines as to when hydrocephalus should be treated, especially in cases in which children are born with dilated ventricles. A better mechanistic understanding of the causes of hydrocephalus and its consequences could provide more targets for intervention and improve clinical outcomes by optimizing the timing of available treatments.

ABBREVIATIONS AF = AlexaFluor; ICP = intracranial pressure; IFN = interferon; IL = interleukin; $M S D=$ Meso Scale Discovery; OPC = oligodendrocyte progenitor cell; PBS = phosphate-buffered saline; PND10 = postnatal day 10; TNF = tumor necrosis factor; WT = wild-type.

SUBMITTED October 23, 2019. ACCEPTED December 5, 2019.

INCLUDE WHEN CITING Published online February 7, 2020; DOI: 10.3171/2019.12.PEDS19625. 
Recently, a model of neonatal hydrocephalus that is due to a primary dysfunction of cilia within the brain has been developed. ${ }^{1,36}$ A mutation in the $C c d c 39$ gene that is selectively expressed in the choroid plexus and ependymal cells of the embryonic brain results in early postnatal hydrocephalus, shortened lifespans, astrogliosis, and reduced myelination. ${ }^{1,36}$ Other forms of brain injury that lead to hydrocephalus include trauma, infection, and intracerebral or intraventricular hemorrhage, all of which induce a robust neuroinflammatory reaction consisting of activated microglia, macrophages, and astrocytes. The neuroinflammation caused by these insults likely contributes to the development of hydrocephalus. However, the effects the hydrocephalus itself has on neuroinflammation are not well understood. Therefore, this ciliopathyinduced model provides a unique opportunity to study the effects of hydrocephalus on inflammation, as opposed to inflammation-mediated hydrocephalus. In addition, we previously observed a significant decrease in myelin production in this model, as evidenced by reduced myelin basic protein levels indicating that hydrocephalus induces myelination failure. ${ }^{1}$ Oligodendrocyte lineage cells are exquisitely sensitive to inflammation present in other neurological diseases such as multiple sclerosis and periventricular leukomalacia. ${ }^{15,25,30}$ In these forms of white matter disease, neuroinflammation is considered the primary mediator of oligodendrocyte progenitor cell (OPC) injury. ${ }^{4,6,31}$ In animal studies of CNS injury, OPCs increase in number within injured white matter but do not reconstitute lost myelin. ${ }^{29,38}$ White matter injury in kaolin-induced hydrocephalus models is also correlated with inflammatory burden. ${ }^{42,43}$

Several recent studies have established a link between hydrocephalus and neuroinflammation. Proinflammatory cytokines are increased in the CSF of preterm infants with posthemorrhagic hydrocephalus, ${ }^{14}$ and animal studies of posthemorrhagic hydrocephalus are also associated with an inflammatory response. ${ }^{13}$ A prior study of rats with inherited aqueductal stenosis has shown an inflammatory response throughout the brain. ${ }^{22} \mathrm{CNS}$ inflammation can also worsen the progression of hydrocephalus by inducing CSF overproduction. ${ }^{18}$ However, there is a growing understanding that neuroinflammation is also reparative in the CNS. Much of this work has focused on CNS macrophages that can exhibit dual modalities in CNS injury. Macrophages react to local environmental cues to generate complex, heterogeneous, and often opposing responses. ${ }^{21}$ Classically activated ("M1") macrophages induce proinflammatory responses often associated with tissue injury, whereas alternatively activated ("M2") macrophages generate antiinflammatory or proreparative responses. This has been well-described in spinal cord injury, ${ }^{11,19}$ where progressive white matter injury occurs. ${ }^{5}$ In this current study, we sought to determine if the hydrocephalus led to a proinflammatory macrophage response as exhibited in other forms of CNS injury.

\section{Methods}

\section{Animals}

Mice were generated (born) at Cincinnati Children's
Hospital Medical Center, and studies were approved by the Institutional Animal Care and Use Committee there. Wild-type (WT) littermate animals were used as controls. All tissue used in this study was harvested from postnatal day 10 (PND10) pups that were maintained on a normal light/dark cycle and kept with their mother. The details of this genetic model of hydrocephalus have been recently published elsewhere. ${ }^{1}$ These animals experience shortened lifespans, and rapidly develop ventriculomegaly. At PND10, these animals have reduced myelin production when compared to littermate controls. ${ }^{1}$ For all experiments, animals were euthanized using $\mathrm{CO}_{2}$ and then decapitated. Brains were removed and processed for either immunohistochemical or biochemical endpoints. A total of 14 mice were used in the study.

\section{Immunohistochemistry}

Tissue used for immunohistochemistry was immersion-fixed in $4 \%$ paraformaldehyde for 18 hours at $4{ }^{\circ} \mathrm{C}$, before a series of phosphate-buffered saline (PBS) washes. Brains were then cryoprotected in a $15 \%$ sucrose/PBS solution for 18 hours, prior to final storage in $30 \%$ sucrose/ PBS solution. After bisecting the brain and embedding it in optimal cutting temperature compound, $12-\mu \mathrm{m}$ sagittal sections were collected starting from the midline and directly mounted onto charged slides and stored at $-20^{\circ} \mathrm{C}$ until use. All staining was conducted with the use of Sequenza immunostaining racks (ThermoFisher Scientific). Briefly, endogenous peroxidase activity was quenched with $0.3 \% \mathrm{H}_{2} \mathrm{O}_{2}$ made in methanol (omitted for immunofluorescent staining) before blocking for nonspecific protein binding with either $10 \%$ goat or donkey serum and $0.2 \%$ Triton X-100 in $1 \times$ PBS. All antibodies were diluted in a $1 \times$ PBS solution with $3 \%$ serum and $0.2 \%$ Triton X-100. For bright field microscopy analysis of NG2 (1:250, Millipore, ab5320), the biotinylated goat anti-rabbit secondary antibody (1:200, Vector, BA-1000) was amplified with an avidin-biotin system (ABC Elite kit, Vector), and

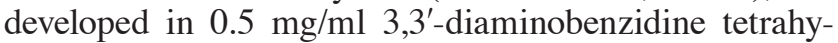
drochloride solution (VWR). The slides were incubated with a nuclear counterstain (Methyl Green, Vector Labs) and dehydrated before being coverslipped with Permount mounting media (Electron Microscopy Sciences). All imaging was performed with a Zeiss Axio Scan.Z1 at magnification $\times 20$.

\section{Immunofluorescence}

Immunofluorescent analyses to evaluate macrophage heterogeneity $\mathrm{y}^{11}$ were completed with images of colabeled rabbit anti-IBA-1 (1:1000, Wako, 019-19741), rat antiCD86 (1:200, BD, 553689), and goat anti-CD206 (1:200, R\&D, AF2535), using AlexaFluor (AF) secondary antibodies donkey anti-rabbit (1:200, AF647, Thermo Scientific, A31573), donkey anti-rat (1:200, AF488, Thermo Scientific, A21208), and donkey anti-goat (1:200, AF546, Thermo Scientific, A11056). Slides were coverslipped using mounting media with DAPI counterstain (Vector Hardset Antifade Mounting Media), and stored at $-20^{\circ} \mathrm{C}$ until imaging. All imaging was performed with a Zeiss Axio Scan.Z1 at magnification $\times 20$. 

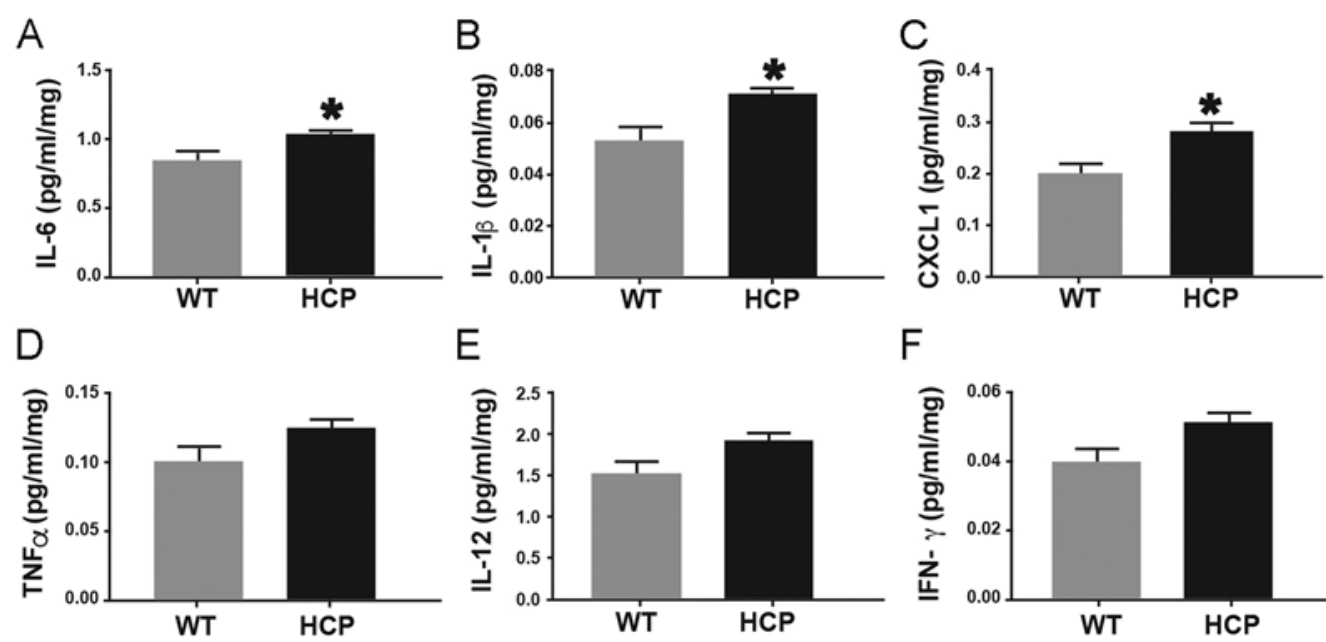

$\mathrm{F}$
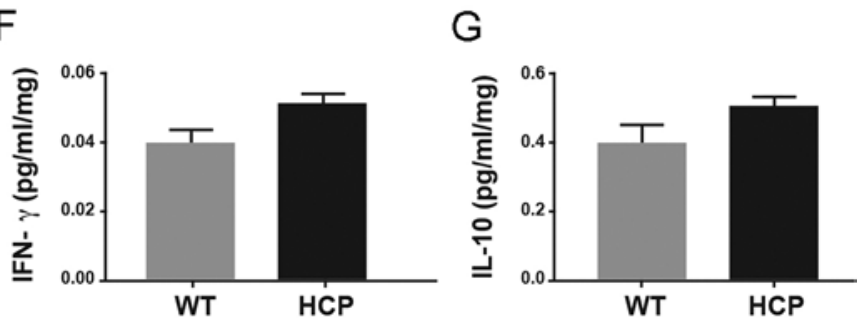

FIG. 1. Overexpression of proinflammatory cytokines in hydrocephalus (HCP) mice. Protein levels of IL-6 (A), IL-1B (B), CXCL1 (C), TNF $\alpha(\mathbf{D})$, IL-12 (E), IFN-y (F), and IL-10 (G) were determined by MSD assay, and values were normalized to total protein. Data are presented as mean \pm SEM ( $n=4$ in each group). ${ }^{*} p<0.05$ vs WT.

\section{Quantitative Analysis of Immunohistochemistry and Immunofluorescence}

Semiautomated, quantitative analysis was performed for both immunohistochemical and immunofluorescent scanned slides using HALO software (version 2.2, Indica Labs). All analysis was performed by a technician blinded to experimental groups. The corpus callosum of sagittal sections was delineated for analysis and thresholds for positive staining set for use across all slides stained under the same conditions. Then, automated algorithms were used to identify and count positively stained cells. The software package generates 2D measurements of areas traced by the user. Positive cells were counted as areas of colocalization of either NG2 or IBA1 with their respective nuclear stains. Cells had to meet other user-set parameters including size limits and intensity thresholds that were applied uniformly across experimental groups. Tissue area and number of positive cells were calculated, and values were expressed as cellular density (cells per $\mathrm{mm}^{2}$ ). These values were presented as fold increase from WT control. The extent of vacuolation was calculated by dividing the area of absent tissue within the corpus callosum by the total area of the corpus callosum traced manually.

\section{Enzyme-Linked Immunosorbent Assay}

Tissue for biochemical endpoints was flash frozen in liquid nitrogen and stored at $-80^{\circ} \mathrm{C}$. Brain hemisphere homogenates were made by shearing tissue with an Omni $\mathrm{TH}$ homogenizer in a PBS lysis buffer with $0.5 \mathrm{mM}$ EDTA, $1 \mathrm{mM}$ phenylmethylsulfonyl fluoride, and protease inhibitors (Pierce). ${ }^{3}$ Levels of interleukin (IL)-1 $\beta$, IL12, interferon (IFN)- $\gamma$, IL-6, CXCL1, IL-10, and tumor necrosis factor (TNF) $-\alpha$ were measured in approximately $50 \mu \mathrm{g}$ of protein using an ultra-sensitivity enzyme-linked immunosorbent assay (ELISA) kit from Meso Scale Discovery (MSD) according to the manufacturer's instructions (MSD Mouse ProInflammatory 7-Plex, N75012B).

\section{Statistical Analysis}

Statistics were computed using Prism software (GraphPad). The Student t-test was used for all analyses. All results are expressed as means \pm SEM. Statistical significance was set at $\mathrm{p}<0.05$.

\section{Results}

\section{Hydrocephalus and Expression of Proinflammatory Cytokines}

To determine the extent to which proinflammatory activation occurs, we measured levels of several cytokines throughout the entire brain of PND10 mice with hydrocephalus and littermate controls. Consistent with histological results, both pro- and antiinflammatory cytokines increased with hydrocephalus, however, proinflammatory cytokines were significantly elevated. Levels of IL-6 (Fig. 1A), IL-1 $\beta$ (Fig. 1B), and CXCL1 (Fig. 1C) were statistically significant, while levels of TNFa (Fig. 1D), IL-12 (Fig. 1E), and IFN- $\gamma$ (Fig. 1F) trended higher than those of control animals. The antiinflammatory cytokine IL-10 was increased in animals with hydrocephalus, but not to a statistically significant degree (Fig. 1G).

\section{Hydrocephalus and Macrophages Within the Corpus Callosum}

IBA1, which labels both resident microglia and infiltrating macrophages, was significantly increased in animals with hydrocephalus within the corpus callosum (Fig. 2). Importantly, this increase was not due to increased corpus callosum size, as the density of IBA-1 labeling increased with hydrocephalus. In control animals, there was an average of $13 \pm 5$ macrophages per $\mathrm{mm}^{2}$ in the corpus callosum, while there were $86 \pm 20$ macrophages per $\mathrm{mm}^{2}$ in hydrocephalic brains (Fig. 2E).

To determine if macrophages were activated toward a proinflammatory versus proregenerative or regulatory phenotype, we labeled tissue with CD86 and CD206, 

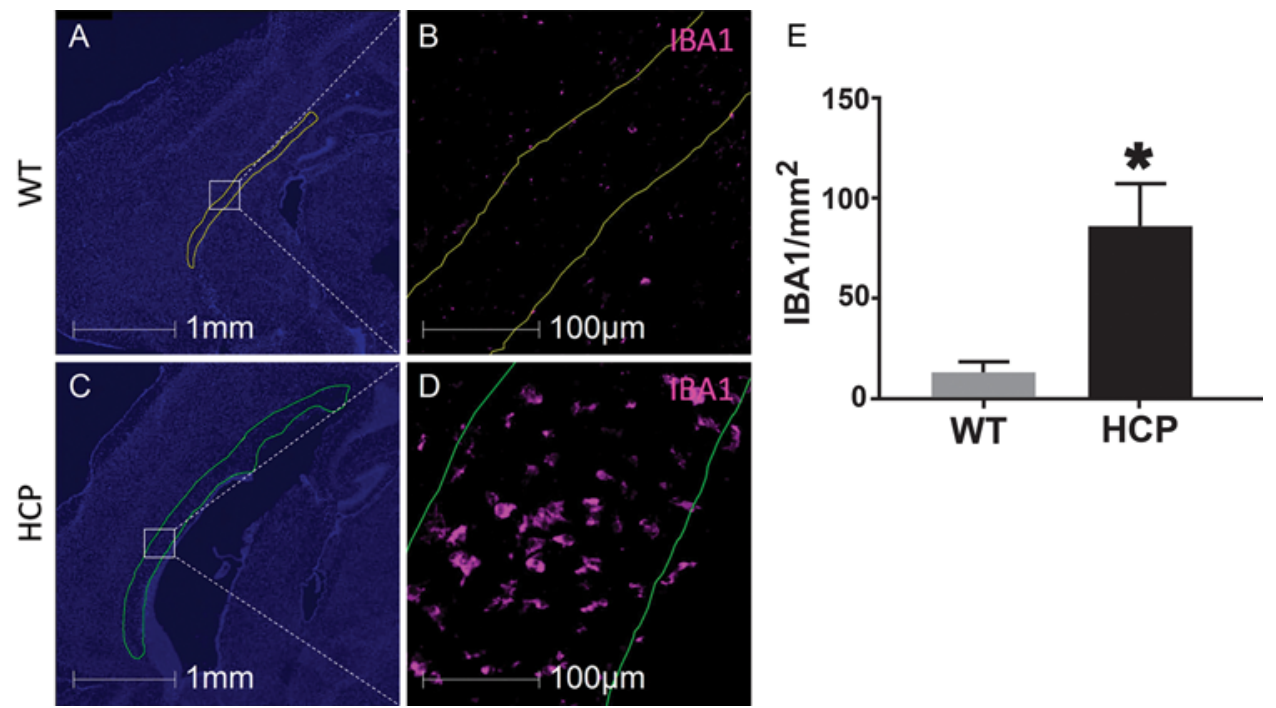

FIG. 2. Increased IBA1-positive cells in the corpus callosum of hydrocephalic animals. Sagittal sections of a WT (A and B) and hydrocephalic (C and D) brain with the corpus callosum outlined. Sections were labeled with DAPI (blue) and IBA1 (pink). IBA1 quantification $(E)$ shows a significant increase in macrophages in the corpus callosum in animals with hydrocephalus. Data are presented as mean \pm SEM ( $n=3$ in each group). * $p<0.05$ vs WT.

markers of proinflammatory macrophage phenotype and proregenerative macrophage phenotype, respectively. ${ }^{19}$ In animals with hydrocephalus, CD86 labeling increased 215- \pm 39-fold while CD206 labeling increased 55- \pm 8-fold (Fig. 3) compared to littermate controls.

\section{Hydrocephalus and Corpus Callosum Morphology}

Prior work has already demonstrated significant ventriculomegaly in this mouse model of hydrocephalus ${ }^{1}$ that was visible in our specimens. We compared the size of the corpus callosum in sagittal sections of hydrocephalic ani- mals (Fig. 4C and D) to WT littermates (Fig. 4A and B) at PND10. There was a visible increase in total size of the corpus callosum in animals with hydrocephalus, although this was not statistically significant (overall cross-sectional area measured $1.49 \pm 0.69 \mathrm{~mm}^{2}$ in hydrocephalic animals versus $0.092 \pm 0.0037 \mathrm{~mm}^{2}$ in controls, $p=0.11$, Fig. 4E). There was also obvious tissue structural disruption present (Fig. 4D). An increase in corpus callosum size was accompanied by a vacuolation of the tissue, with significant tissue loss. Animals with hydrocephalus had approximately 2.5-fold more loss of tissue within the corpus callosum (Fig. 4F).
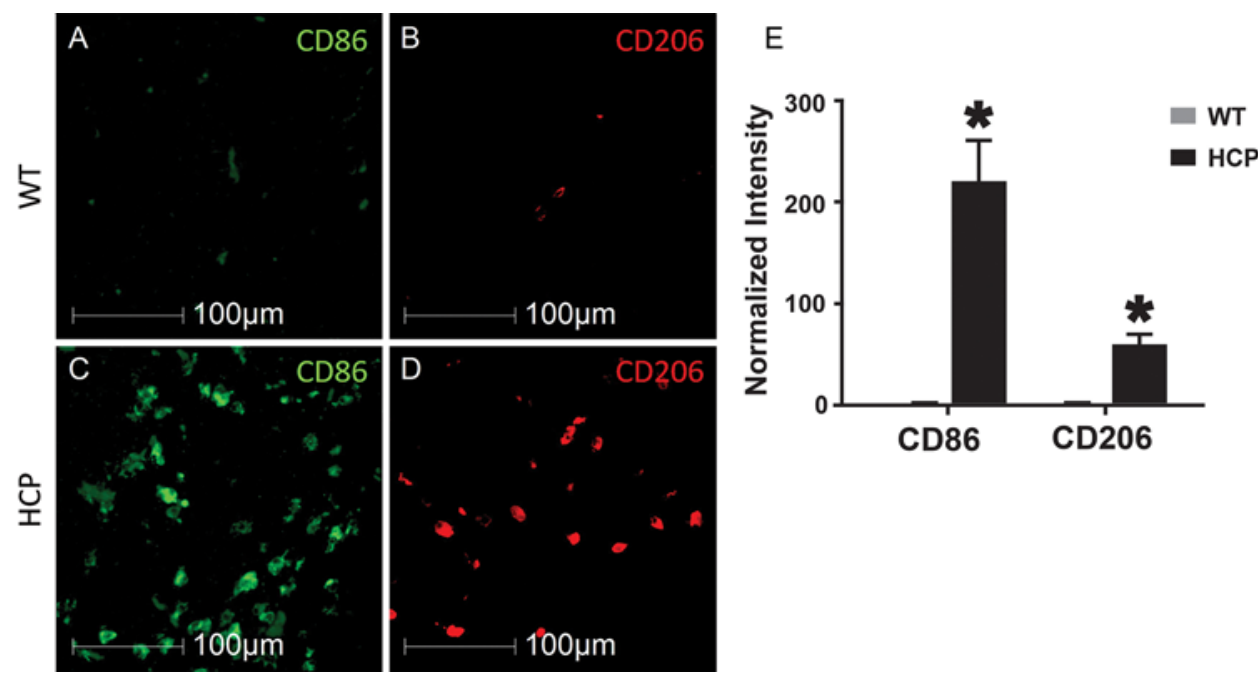

FIG. 3. Macrophage polarization in the corpus callosum. Pro- (M1) and antiinflammatory (M2) macrophages are labeled in control (A and B) and hydrocephalic (C and D) animals. M1 (CD86; A and C) versus M2 (CD206; B and D) macrophages in the corpus callosum are shown as measured with quantitative immunohistochemistry $(E)$. Data are presented as mean \pm SEM $(n=3$ in each group). ${ }^{*} p<0.05$ vs WT. 


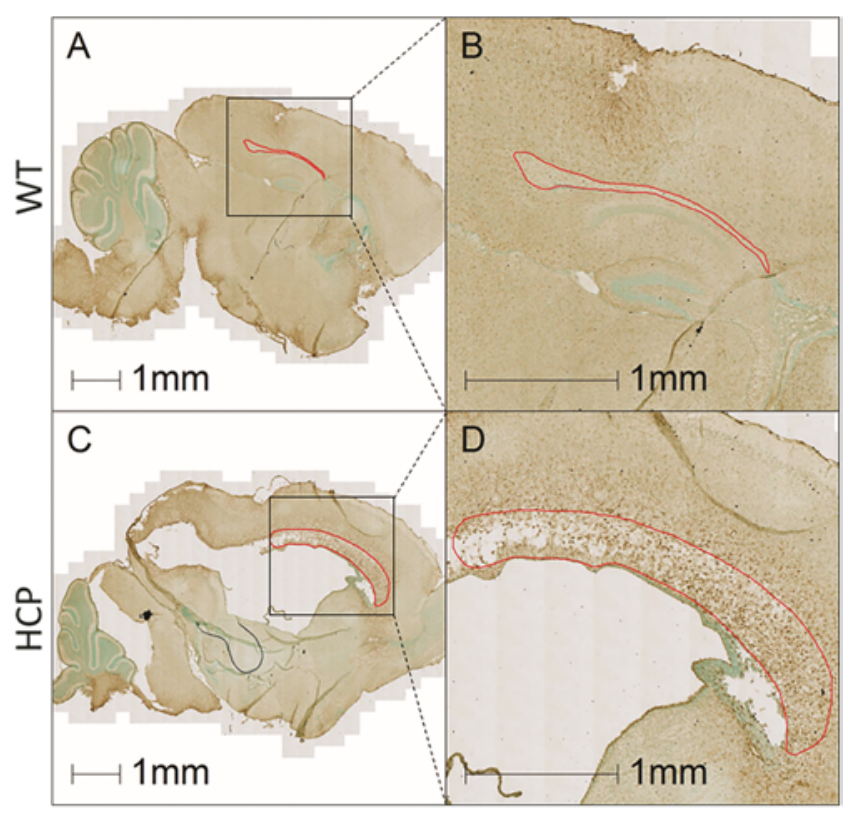

$\mathrm{E}$
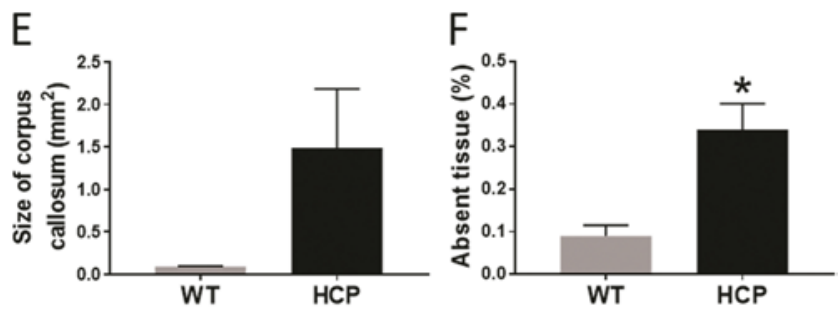

FIG. 4. Corpus callosum enlargement and vacuolation in Ccdc39-mutant mice compared to WT animals. Sagittal sections of PND10 WT (A) and hydrocephalic (C) mice stained with NG2 (brown) and methyl green (green). Higher magnification outline of the corpus callosum (red outline) in WT (B) and hydrocephalic (D) animals shows increased size and vacuolation in the corpus callosum in hydrocephalus. Analysis of size (E) and lost tissue (F) in the corpus callosum demonstrates an increase in corpus callosum volume (not significant, $p=0.11$ ) but concomitant significant tissue loss. Data are presented as mean \pm SEM $(n=3$ in each group). ${ }^{*} p<0.05$ vs WT.

\section{Hydrocephalus and Upregulation of NG2}

NG2 is a proteoglycan expressed in OPCs and often used as a marker for immature, promyelinating oligodendrocyte lineage cells. However, it is also expressed on pericytes ${ }^{35}$ and is upregulated in other forms of brain injury. ${ }^{23} \mathrm{NG} 2$ in the corpus callosum increased significantly in animals with hydrocephalus, with $1331 \pm 53$ NG2 cells/ $\mathrm{mm}^{2}$ (Fig. 5C and D), compared to littermate controls with $693 \pm 178$ NG2 cells $/ \mathrm{mm}^{2}$ (Fig. 5A, B, and E).

\section{Discussion}

The etiologies of hydrocephalus are diverse and include intraventricular hemorrhage, infection, structural abnormalities such as aqueductal stenosis, and genetic causes such as $L 1 C A M$ mutation. ${ }^{17}$ Translational animal studies have examined various models of hydrocephalus, and focus on the predominant cause in developed nations, i.e., intraventricular hemorrhage. ${ }^{41}$ Hemorrhagic and infectious causes of hydrocephalus are associated with inflammation in animal models and human studies, ${ }^{14,33,37}$ however, there is little understanding as to whether or not hydrocephalus itself causes neuroinflammation. This animal model of hydrocephalus due to ciliopathy from a $C c d c 39$ mutation has the advantage of having no antecedent proinflammatory insult, therefore inflammatory changes present can be interpreted as the result of increased CSF pressure and not an inciting inflammatory stimulus. The use of the term "hydrocephalus" rather than "ventriculomegaly" generally implies elevated intracranial pressure (ICP), which can be highly variable between different etiologies and severities of disease. While this mouse model does not provide the opportunity to verify elevated ICP, the combination of altered CSF circulation, ${ }^{1}$ coupled with ventriculomegaly and domed head shape, ${ }^{36}$ leads us to classify this pathology as hydrocephalus.

Microglia, the resident macrophage of the brain, are the main mediators of neonatal neuroinflammation and white matter injury. ${ }^{7,9}$ Microglia are activated by many different stimuli in CNS injury and disease, including extracellular iron that plays a role in posthemorrhagic hydrocephalus. ${ }^{44}$ We hypothesized that macrophage activation causes white matter loss even without hemorrhage in this animal model. ${ }^{1}$ Our results, that macrophage activation occurs in hydrocephalus without antecedent hemorrhage or infection, demonstrate that increased CSF and ICP alone may be sufficient to activate macrophages..$^{20,40}$ Conversely, it is possible that macrophages are activated as a consequence of structural injury to white matter that occurs due to increased ICP. It is also possible that a feed-forward loop occurs, in which hydrocephalus directly injures white matter and activates macrophages, which further injure white matter. Another possibility is that increased ICP leads to reduced white matter perfusion, and that the inflammation we observe is a downstream effect of tissue ischemia. Indeed, there is evidence to suggest that even low-pressure hydrocephalus can lead to a reduction in white matter perfusion. ${ }^{45}$

Our finding of robust inflammation within the corpus callosum in the setting of hydrocephalus is in contrast to another recent study in a genetic model of hydrocephalus due to an LICAM mutation in which there was no astrogliosis or microglial activation observed. ${ }^{8}$ The contrast between these data may be due to the severity and time course of hydrocephalus development, as L1CAM-mutant rats develop hydrocephalus over a more prolonged course than the $C c d c 39$ mutant mice in this study. Future trials of CSF diversion or antiinflammatory therapy in translational hydrocephalus models will help determine the causal relationships between hydrocephalus, inflammation, and white matter injury.

Although macrophages can mediate white matter injury, they also play a role in recovery and remodeling of the injured CNS. ${ }^{12,34}$ While macrophages with heterogeneous properties migrate into the CNS acutely after both traumatic spinal cord and brain injury, proinflammatory macrophages predominate over antiinflammatory macrophages chronically. ${ }^{16}$ Although controversial, the spectrum of activation and function is sometimes simplified by designating proinflammatory macrophages as "M1" and antiinflammatory as "M2."'12,27 We used immunohis- 


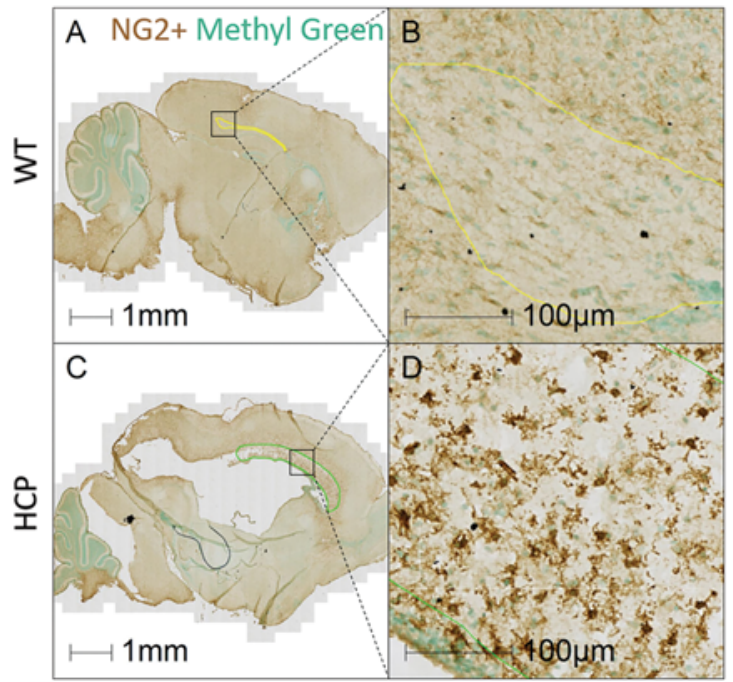

$\mathrm{E}$

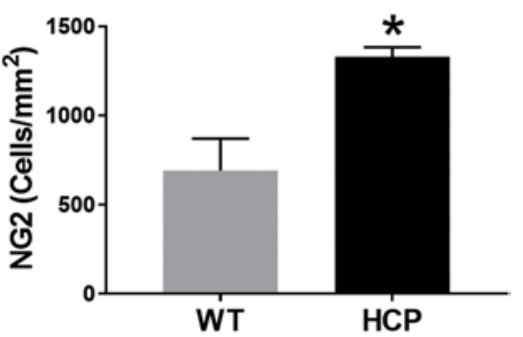

FIG. 5. Increased NG2 cells in the corpus callosum of hydrocephalic animals as compared to WT animals. Sagittal sections of PND10 WT (A and B) and hydrocephalic (C and D) mice stained with NG2 (brown) and methyl green (green) for nuclei. NG2 density in the corpus callosum significantly increased in animals with hydrocephalus (E). Data are presented as mean $\pm S E M(n=3$ in each group). ${ }^{*} p<0.05$ vs WT.

tochemical markers associated with M1 and M2 activation to help better determine if macrophage activation in hydrocephalus followed patterns seen in neurotrauma of a predominant M1 phenotype. We found significant increases in proinflammatory cytokines IL-6 (Fig. 1A) and IL-1 $\beta$ (Fig. 1B) in mice with hydrocephalus. Our results indicate a polarization of M1 versus M2 activation just as in traumatic injuries, and demonstrate not only macrophage activation but a shift toward a more inflammatory macrophage phenotype as opposed to a reparative one. The data reported here cannot discern the origin of macrophages-microglia or infiltrating macrophages-in this model, but future work with inhibitors of peripheral macrophage infiltration ${ }^{26}$ could help define the origin of activated macrophages in hydrocephalus.

NG2 proteoglycan is a marker of immature, premyelinating OPCs, ${ }^{24,28}$ but is also found on other cells in the CNS. ${ }^{2}$ Our data demonstrate increased NG2 cells within the corpus callosum of animals with hydrocephalus. Although this may seem counterintuitive in this model where decreased myelination occurs, it is consistent with results seen in traumatic brain and spinal cord injury. ${ }^{38,39} \mathrm{NG} 2$ is upregulated by proinflammatory cytokines and participates in formation of the glial scar found in CNS injury. ${ }^{32}$ Therefore, the increase in NG2-positive cells correlated with our other findings of an inflammatory response within white matter.

\section{Conclusions}

Our data support an association between macrophage activation and white matter injury in hydrocephalus, similar to findings from other disease models. Because this model does not involve a proinflammatory stimulus, such as hemorrhage, it is possible that the activation of microglia by CSF accumulation alone induces macrophage activation and gliosis in the corpus callosum. While the short lifespan of animals in this model limits our ability to conduct long-term outcome studies and behavioral analyses, additional studies using antiinflammatory therapies or CSF diversion could help determine the potential of different therapeutic strategies to reduce inflammatory white matter injury in hydrocephalus. Future studies could also examine other brain structures such as the hippocampus that are affected by other causes of hydrocephalus. ${ }^{10}$

\section{Acknowledgments}

This publication is based upon work supported in part by the University of Kentucky CCTS KL2 program to B.A.M. and University of Kentucky CCTS Young Investigator Pilot Award no. UL1TR001998 to B.A.M. and J.C.G.

\section{References}

1. Abdelhamed Z, Vuong SM, Hill L, Shula C, Timms A, Beier $\mathrm{D}$, et al: A mutation in Ccdc39 causes neonatal hydrocephalus with abnormal motile cilia development in mice. Development 145:145, 2018

2. Ampofo E, Schmitt BM, Menger MD, Laschke MW: The regulatory mechanisms of NG2/CSPG4 expression. Cell Mol Biol Lett 22:4, 2017

3. Bachstetter AD, Rowe RK, Kaneko M, Goulding D, Lifshitz J, Van Eldik LJ: The p38 $\alpha$ MAPK regulates microglial responsiveness to diffuse traumatic brain injury. J Neurosci 33:6143-6153, 2013

4. Back SA: White matter injury in the preterm infant: pathology and mechanisms. Acta Neuropathol 134:331-349, 2017

5. Crowe MJ, Bresnahan JC, Shuman SL, Masters JN, Beattie MS: Apoptosis and delayed degeneration after spinal cord injury in rats and monkeys. Nat Med 3:73-76, 1997

6. Dendrou CA, Fugger L, Friese MA: Immunopathology of multiple sclerosis. Nat Rev Immunol 15:545-558, 2015

7. Denker SP, Ji S, Dingman A, Lee SY, Derugin N, Wendland $\mathrm{MF}$, et al: Macrophages are comprised of resident brain mi- 
croglia not infiltrating peripheral monocytes acutely after neonatal stroke. J Neurochem 100:893-904, 2007

8. Emmert AS, Vuong SM, Shula C, Lindquist D, Yuan W, Hu YC, et al: Characterization of a novel rat model of X-linked hydrocephalus by CRISPR-mediated mutation in L1cam. J Neurosurg 132:945-958, 2020

9. Ernst LM, Gonzalez J, Ofori E, Elovitz M: Inflammationinduced preterm birth in a murine model is associated with increases in fetal macrophages and circulating erythroid precursors. Pediatr Dev Pathol 13:273-281, 2010

10. Garton TP, He Y, Garton HJ, Keep RF, Xi G, Strahle JM: Hemoglobin-induced neuronal degeneration in the hippocampus after neonatal intraventricular hemorrhage. Brain Res 1635:86-94, 2016

11. Gensel JC, Kopper TJ, Zhang B, Orr MB, Bailey WM: Predictive screening of M1 and M2 macrophages reveals the immunomodulatory effectiveness of post spinal cord injury azithromycin treatment. Sci $\operatorname{Rep~7:40144,~} 2017$

12. Gensel JC, Zhang B: Macrophage activation and its role in repair and pathology after spinal cord injury. Brain Res 1619:1-11, 2015

13. Gram M, Sveinsdottir S, Ruscher K, Hansson SR, Cinthio M, Akerström B, et al: Hemoglobin induces inflammation after preterm intraventricular hemorrhage by methemoglobin formation. J Neuroinflammation 10:100, 2013

14. Habiyaremye G, Morales DM, Morgan CD, McAllister JP, CreveCoeur TS, Han RH, et al: Chemokine and cytokine levels in the lumbar cerebrospinal fluid of preterm infants with post-hemorrhagic hydrocephalus. Fluids Barriers CNS 14:35, 2017

15. Jensen FE: The role of glutamate receptor maturation in perinatal seizures and brain injury. Int J Dev Neurosci 20:339347,2002

16. Jin X, Ishii H, Bai Z, Itokazu T, Yamashita T: Temporal changes in cell marker expression and cellular infiltration in a controlled cortical impact model in adult male C57BL/6 mice. PLoS One 7:e41892, 2012

17. Kahle KT, Kulkarni AV, Limbrick DD Jr, Warf BC: Hydrocephalus in children. Lancet 387:788-799, 2016

18. Karimy JK, Zhang J, Kurland DB, Theriault BC, Duran D, Stokum JA, et al: Inflammation-dependent cerebrospinal fluid hypersecretion by the choroid plexus epithelium in posthemorrhagic hydrocephalus. Nat Med 23:997-1003, 2017

19. Kigerl KA, Gensel JC, Ankeny DP, Alexander JK, Donnelly DJ, Popovich PG: Identification of two distinct macrophage subsets with divergent effects causing either neurotoxicity or regeneration in the injured mouse spinal cord. J Neurosci 29:13435-13444, 2009

20. Lan X, Han X, Li Q, Yang QW, Wang J: Modulators of microglial activation and polarization after intracerebral haemorrhage. Nat Rev Neurol 13:420-433, 2017

21. Lawrence T, Natoli G: Transcriptional regulation of macrophage polarization: enabling diversity with identity. Nat Rev Immunol 11:750-761, 2011

22. Mangano FT, McAllister JP II, Jones HC, Johnson MJ, Kriebel RM: The microglial response to progressive hydrocephalus in a model of inherited aqueductal stenosis. Neurol Res 20:697-704, 1998

23. Mattugini N, Merl-Pham J, Petrozziello E, Schindler L, Bernhagen J, Hauck SM, et al: Influence of white matter injury on gray matter reactive gliosis upon stab wound in the adult murine cerebral cortex. Glia 66:1644-1662, 2018

24. McDonald JW, Levine JM, Qu Y: Multiple classes of the oligodendrocyte lineage are highly vulnerable to excitotoxicity. Neuroreport 9:2757-2762, 1998

25. Miller BA, Crum JM, Tovar CA, Ferguson AR, Bresnahan JC, Beattie MS: Developmental stage of oligodendrocytes determines their response to activated microglia in vitro. $\mathbf{J}$ Neuroinflammation 4:28, 2007
26. Morganti JM, Jopson TD, Liu S, Riparip LK, Guandique CK, Gupta N, et al: CCR2 antagonism alters brain macrophage polarization and ameliorates cognitive dysfunction induced by traumatic brain injury. J Neurosci 35:748-760, 2015

27. Morganti JM, Riparip LK, Rosi S: Call off the $\operatorname{dog}(\mathrm{ma})$ : M1/ M2 polarization is concurrent following traumatic brain injury. PLoS One 11:e0148001, 2016

28. Nishiyama A, Boshans L, Goncalves CM, Wegrzyn J, Patel KD: Lineage, fate, and fate potential of NG2-glia. Brain Res 1638 (Pt B): 116-128, 2016

29. Ortega SB, Kong X, Venkataraman R, Savedra AM, Kernie SG, Stowe AM, et al: Perinatal chronic hypoxia induces cortical inflammation, hypomyelination, and peripheral myelinspecific T cell autoreactivity. J Leukoc Biol 99:21-29, 2016

30. Pang Y, Cai Z, Rhodes PG: Effect of tumor necrosis factor- $\alpha$ on developing optic nerve oligodendrocytes in culture. $\mathbf{J}$ Neurosci Res 80:226-234, 2005

31. Rezaie P, Dean A: Periventricular leukomalacia, inflammation and white matter lesions within the developing nervous system. Neuropathology 22:106-132, 2002

32. Rhodes KE, Raivich G, Fawcett JW: The injury response of oligodendrocyte precursor cells is induced by platelets, macrophages and inflammation-associated cytokines. Neuroscience 140:87-100, 2006

33. Rohlwink UK, Mauff K, Wilkinson KA, Enslin N, Wegoye E, Wilkinson RJ, et al: Biomarkers of cerebral injury and inflammation in pediatric tuberculous meningitis. Clin Infect Dis 65:1298-1307, 2017

34. Schwartz M, Lazarov-Spiegler O, Rapalino O, Agranov I, Velan G, Hadani M: Potential repair of rat spinal cord injuries using stimulated homologous macrophages. Neurosurgery 44:1041-1046, 1999

35. Stallcup WB: The NG2 proteoglycan in pericyte biology. Adv Exp Med Biol 1109:5-19, 2018

36. Stottmann RW, Moran JL, Turbe-Doan A, Driver E, Kelley M, Beier DR: Focusing forward genetics: a tripartite ENU screen for neurodevelopmental mutations in the mouse. Genetics 188:615-624, 2011

37. Strahle J, Garton HJ, Maher CO, Muraszko KM, Keep RF, $\mathrm{Xi} \mathrm{G}$ : Mechanisms of hydrocephalus after neonatal and adult intraventricular hemorrhage. Transl Stroke Res 3 (Suppl 1):25-38, 2012

38. Sun F, Lin CL, McTigue D, Shan X, Tovar CA, Bresnahan JC, et al: Effects of axon degeneration on oligodendrocyte lineage cells: dorsal rhizotomy evokes a repair response while axon degeneration rostral to spinal contusion induces both repair and apoptosis. Glia 58:1304-1319, 2010

39. Susarla BT, Villapol S, Yi JH, Geller HM, Symes AJ: Temporal patterns of cortical proliferation of glial cell populations after traumatic brain injury in mice. ASN Neuro 6:159-170, 2014

40. Ulfig N, Bohl J, Neudörfer F, Rezaie P: Brain macrophages and microglia in human fetal hydrocephalus. Brain Dev 26:307-315, 2004

41. Wright Z, Larrew TW, Eskandari R: Pediatric hydrocephalus: current state of diagnosis and treatment. Pediatr Rev 37:478-490, 2016

42. Yuan W, Deren KE, McAllister JP II, Holland SK, Lindquist DM, Cancelliere A, et al: Diffusion tensor imaging correlates with cytopathology in a rat model of neonatal hydrocephalus. Cerebrospinal Fluid Res 7:19, 2010

43. Yuan W, McAllister JP II, Lindquist DM, Gill N, Holland SK, Henkel D, et al: Diffusion tensor imaging of white matter injury in a rat model of infantile hydrocephalus. Childs Nerv Syst 28:47-54, 2012

44. Zhang Z, Zhang Z, Lu H, Yang Q, Wu H, Wang J: Microglial polarization and inflammatory mediators after intracerebral hemorrhage. Mol Neurobiol 54:1874-1886, 2017

45. Ziegelitz D, Arvidsson J, Hellström P, Tullberg M, Wik- 
kels $\varnothing$ C, Starck G: Pre-and postoperative cerebral blood flow changes in patients with idiopathic normal pressure hydrocephalus measured by computed tomography (CT)-perfusion. J Cereb Blood Flow Metab 36:1755-1766, 2016

\section{Disclosures}

The authors report no conflict of interest concerning the materials or methods used in this study or the findings specified in this paper.

\section{Author Contributions}

Conception and design: Miller, Goulding, Mangano. Acquisition of data: Miller, Goulding, Vogel, Shula, Goto. Analysis and interpretation of data: Miller, Goulding, Pandya, Gensel. Drafting the article: Miller, Pandya, Gensel. Critically revising the article: Miller, Goulding, Shula, Gensel, Mangano, Goto. Reviewed submitted version of manuscript: all authors. Approved the final version of the manuscript on behalf of all authors: Miller. Statistical analysis: Miller, Gensel. Study supervision: Miller.

\section{Correspondence}

Brandon A. Miller: University of Kentucky, Lexington, KY. brandonmiller@uky.edu. 